\title{
A MODERN COURSE ON DIGITAL DESIGN AT THE UNIVERSITY OF TWENTE
}

\author{
Dr. Martin F. Beusekamp \\ University of Twente \\ Department of Computer Science \\ P.O. Box 217 \\ 7500 AE Enschede \\ The Netherlands \\ telephone: $+3153893796 /+3153893770$ \\ fax: +3153309723 \\ e-mail: beusekam@cs.utwente.nl
}

\begin{abstract}
At the University of Twente, a modern course on basic digital design has been developed. This course, which is based on a text book in English[1], is supported by a sophisticated Computer Assisted Learning program and life-like practical work with extensive use of Computer Aided Design tools. These features largely reduce the nced for human teachers. Major parts of the course are particularly suited to be copied by other institutes.
\end{abstract}

\section{INTRODUCTION}

The University of Twente is a medium-sized university for technical and social sciences in the east of The Netherlands. Part of the curriculum for all firstyear's students in Computer Science or Electrical Engineering is a course on basic digital design, which takes the average student approximately 120 hours to complete. Each year, some 250 students attend this course. For over fifteen years, it has consisted of three types of education: classical-style plenary lectures, Computer Assisted Leaming (CAL) and practical work. This formula has proven to be successful over the years. With the aim to update the course to state-of-theart digital design methods and to reduce the need for human support, all parts have been modernized thoroughly during the past few ycars. In this paper, they will be discussed in detail.

\section{SCOPE OF THE COURSE}

The course covers most, but not all topics of the text book, "Digital Design Fundamentals" by K.J. Breeding[1]. Aim of the course is to focus mainly on the synthesis of both combinational and clocked sequential digital systems. Students learn and practise designing at gate/flipflop level as well as at the level of MSI-components like multiplexers, registers and counters. Also, extensive attention is given to the analysis of digital systems as this can provide a better insight into the specific problems that may arise in the design process. Students who attend this course have no digital background yet. Therefore, at the beginning of the course, general theoretical topics like Boolean algebra, number systems, radix conversion, binary and complement arithmetic and coding are covered as well.

\section{LECTURES}

All of the theory in the course is presented to the students by experienced teachers in a total of 12 plenary sessions of 90 minutes cach. These lectures are not compulsory. They are generally attended by $70-80 \%$ of the complete population. Although the lectures are intended to be a discussion platform rather than a teachers' monologue and the students are encouraged to participate actively, the large number of students attending the lectures prevents a lot of personal attention. The text book, however, is written in clear English with many examples and is very well suited for self-study. In retrospect, many students state that they could have completed the course without the lectures.

\section{COMPUTER ASSISTED LEARNING}

\section{General}

To become more familiar with the matter to be studied, students use a Computer Assisted Learning (CAL) program, which is compulsory. This CALprogram was developed by the Educational Centre of 
the University of Twente, together with the teachers of the course[2][3]. It is still subject to extension and improvement. The course is written in a new and modern authoring language called Inigo ${ }^{1}$ and runs in Microsoft's Windows ${ }^{\mathrm{TM}, 2}$ environment. All CALexercises are designed to match the contents of the text book. However, as general topics are covered, with only minor adaptations, the CAL-program could be used with other books. An English version will soon be available.

The total CAL-program consists of over 30 different exercises, covering most of the basic skills and knowledge which are taught in the course. A student has complete control over the program and is able to switch from one exercise to another or to stop practising at any moment. All exercise problems are generated (quasi-)randomly, with a sufficient total number of possibilities to ensure a very low probability of the same problem being presented to two or more students. There are two modes of operation, the exercise mode and the test mode. In exercise mode, the computer gives adequate feedback on all possible types of mistakes a student might make. Also, a lot of positive feedback is given when no mistakes are being made to encourage the student. In test mode, the feedback is minimized. The student now has to show his/her ability to solve a problem without help. This has to be done for all types of problems. A simple good/fault judgement is given by the computer, sometimes allowing for one minor mistake. As no trivial problems are generated by the computer, a student can run a problem in test mode as often as (s)he likes and is also allowed to relum to exercise mode between tests for an unlimited number of times. The good/fault judgement on all problems is filed by the computer. At any time, a student can get an overview of his/her score so far. A sufficient number of computers is available during 64 hours per week, basically all year round.

\section{An example of $\mathrm{CAL}$}

Eight of the exercises in the CAL-program deal with the use of basic flipflops. In four of them, the student is able to practise with the specifications of D-, JK-, T- and SR-flipflops (i.e. given certain input signals, which action does the flipflop perform?). This is part of the logic analysis of the flipflops. In the other four exercises, the student practises designing with the same types of flipflops (i.e. given a desired action, which input signals should be applied to the flipflop?). This is logic synthesis using flipflops.

Figure 1 shows a typical screen that a student could meet while designing with JK-flipflops. In this specific exercise, the student is asked to derive the $\mathrm{J}$ and $\mathrm{K}$-input signals for two JK-flipflops in order to reach a given new state $Q_{2} Q_{1}$ from a given current state $q_{2} q_{1}$. First, it should be noted that figure 1 shows a common Windows ${ }^{\mathrm{TM}}, 2$ screen with a coloured header showing the title of the active program and buttons to close, minimize and maximize the screen. The menu under the header is typical for all CAL-exercises. With the option "options", the student can choose between the above mentioned exercise mode and test mode. With the option "info", the student can either get the requirements to pass the test on the present exercise or get an overview of the score on all exercises until the present moment.

For the sake of clarity, the main part of the screen, under the menu, has the same grouping of items in all exercises.

In the upper left-hand corner, the problem to.be solved is stated. This rectangle contains the same information (question) as long as the given problem has not been solved completely. Thereby, a student is never forced to toggle between screens whilc exercising. This has been a major policy in the design and implementation of the CAL-program.

In the upper right-hand corner, some useful information may be given. Students are allowed to use any written material, for instance the text book, while exercising with the CAL-program. In fact, in a CALsituation with no teacher present, the use of text books cannot be avoided in the first place. In this particular case, the present-state/next-state truth table of a JKflipflop is given to save the student the trouble of looking it up in the text book. In other situations, where no additional information is required, this rectangle may be omitted.

The rectangle in the lower right-hand comer is the student's work space. Here, the student gives answers to the questions in the upper left-hand corner by pointing the mouse arrow at the desired field and clicking the mouse button. In this example, the required $\mathrm{J}$ - and $\mathrm{K}$-input signals can be toggled between 0,1 and - (don't care) with the mouse button. The "ready"-button serves as an enter-key to let the computer know that, to the student's opinion, an answer is complete.

The rectangle in the lower left-hand comer is blank at the beginning of the exercise and shows to-the-point feedback as soon as the student has given an answer. As possible answers are always simple (in this case 0,1 or - , the feedback covers all possible mistakes and, of course, the proper answer. This too, is a major policy in the CAL-program. After three failures by the student, the computer will give the correct answer.

In the example of figure 1, the student has correctly solved the first two state transitions (from 
$q_{2} q_{1}=00$ to $Q_{2} Q_{1}=11$ and from $q_{2} q_{1}=11$ to $Q_{2} Q_{1}=10$ ) within three attempts. At the end of these two partial problems, the feedback which was given while exercising, was summarized in a " +" sign, which is green on the actual screen, to indicate that these two state-transition problems were solved correctly. In the next two partial problems, the student has obviously made too many mistakes, as indicated by the "-" signs, which are red on the actual screen. The feedback in the lower left-hand comer is the last feedback on the last $\left(q_{2} q_{1}=11\right.$ to $\left.Q_{2} Q_{1}=11\right)$ transition problem, after which the correct answer was given. Immediately, the computer quasi-randomly generates a new problem, in this case a $q_{2} q_{1}=11$ to $Q_{2} Q_{1}=00$ transition, and is ready to accept a new answer.

By the easy mouse operations, extensive communication with the computer is avoided and the student is able to concentrate on solving the problem rather than operating the computer. In this way, an exercise like this one will only take a few minutes. As was mentioned before, in test mode no feedback is given and no or only minor student mistakes are accepted.

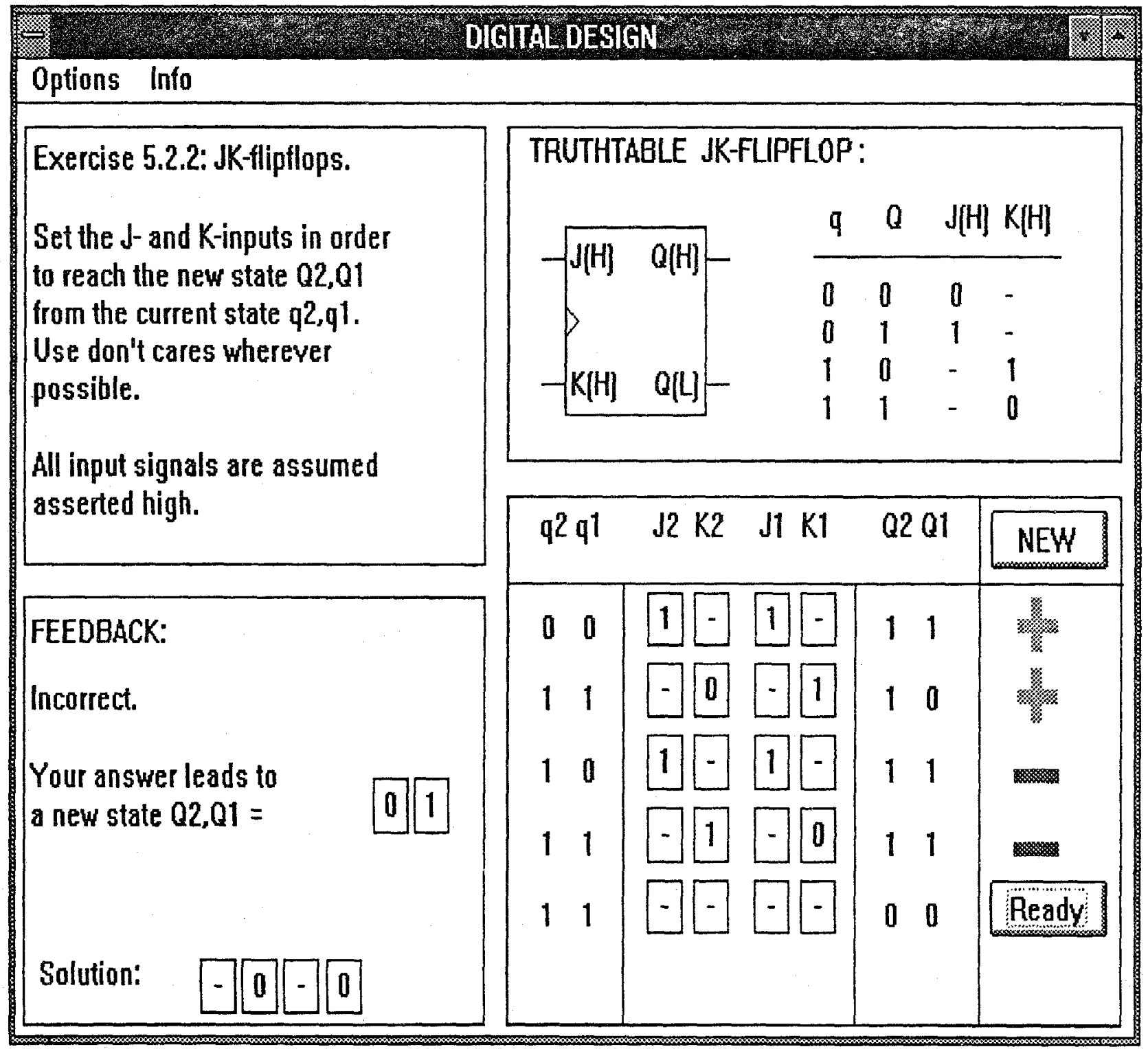

Figure 1. A typical example of a work screen in the Computer Assisted Learning course on Digital Design.

\section{Evaluation of CAL}

In general, students are less reserved to make the mistakes that accompany a lcarning process in front of a computer than in front of a human teacher. In fact, this anonymity is one of the strengths of CAL. However, without violating the anonymity, the computer kept some statistics on test moments, the number of times that a test was taken and so on. Moreover, upon finishing all CAL-tests, students were 
asked to complete a questionnaire. Last year, the results of 270 students were analyzed and cvaluated.

On the average, students required 7.3 hours to complete all 27 exercises that were operational last year (minimum: 4 hours, maximum: 15 hours, standard deviation: 3.2 hours) in approximately four sessions of CAL. The average number of tests taken by a student before successfully completing one exercise ranged from 1.1 at the simplest binary-to-decimal conversion to 2.5 at a more complex Karnaugh-map minimization problem.

A vast majority of the students experienced CAL as useful and pleasant. In no case, operating the computer was a problem and the constant lay-out of the screen highly contributed to the user-friendliness. Most students indicated that studying and practising with a CAL-program is an efficient and motivating way of learning. The availability of the CAL-program at virtually any desired moment and the ability to work at one's own speed was highly appreciated. Almost $80 \%$ of the students wanted CAL-exercises on topics not yet covered, or even on other courses.

\section{PRACTICAL WORK}

Within the complete course on digital design, a student also has to do a total of 24 hours of practical work. Where the basic skills and knowledge are trained in CAL-program, the goal of the practical work is to go through the whole process of designing and devcloping a moderately complex finite state machine, consisting of approximately 25 states.

A pair of students is given a specification of a practical machine in a natural language. Typical examples of these machines are a traffic light controller or a burglar alarm. The first sicp is to transform this informal specification into a formal specification, in our case a state diagram. This has to be done by hand as no computer is able yet 10 interpret a natural language adequately enough for this purpose. Then, the formal specification is fed into a personal computer, where a design and simulation tool called $\mathrm{LOG} / \mathrm{iC}^{3}$ helps to complete the design. Another computer tool, called USE ${ }^{4}$, provides a graphical interface to the user. The design is simulated and improved by the student until, in simulation, it functions correctly. Then, $\mathrm{LOG} / \mathrm{iC}^{3}$ provides a so-called JEDEC-file, which can directly be fed into an Erasable Programmable Logic Device (EPLD) programmer. An actual integrated circuit is then programmed and has to be tested in a life-like testing machine. Any failures arising here are obviously failures in interpreting the simulation results of the CAD-tools. In such a case, the EPLD is crased and a new and corrected JEDEC-file has to be produced. Thus, the whole designing process from informal specification to a tested integrated circuit is covered.

\section{SUMMARY AND CONCLUSIONS}

A very modem course on basic digital design has been developed. All materials, i.e. the book, the CALprogram and the computer tools for the practical work are available on the free market for reasonable prices and are supported professionally. Little effort will have to be put into transferring the course from our university to any other in the world. The practical work is very much like every-day industrial work. Most students feel attracted by the CAL-program and the practical work. Examination results are good.

\section{ACKNOWLEDGEMENTS}

The author wishes to thank Gerdy C. ten Bruggencate and Jan T. van der Veen for their inventive implementation of the CAL-course on Digital Design and Tom R.C. Bonnema for our fruitful discussions concerning the contents of this course.

\section{REFERENCES}

[1] Kenneth J. Breeding, Digital Design Fundamentals, $2^{\text {nd }}$ edition. Prentice-Hall International Editions, Englewood Cliffs, New Jersey 07632, 1992. ISBN 0-13-213406-3.

[2] Jan T. van der Veen and Gerdy C. ten Bruggencate, "Computer Ondersteund Onderwijs in Digitale Techniek" (Computer Assisted Education in Digital Techniques), in Proceedings of NIOC'92 Conference, Maastricht, The Netherlands, 1992. In Dutch.

[3] Gerdy C. ten Bruggencate, Jan T. van der Vecn, Martin F. Beusekamp and Tom R.C. Bonnema, "CAL on Digital Design: Student Controlled Practising \& Testing", in Proccedings of the European Conference on Educational Research, Enschede, The Netherlands, 1992.

1) Inigo is a product of the Educational Centre of the University of Twente, Enschede, The Netherlands.

2) Windows is a trademark of Microsoft Corporation.

3) LOG/iC is a product of ISDATA GmbH, Karlsruhe, Germany.

4) USE is a product of Translogic b.v., Enschede, The Netherlands. 